\title{
Quantum Chaos in SU(3) Models with Trapped Ions
}

\author{
Tobias Graß, ${ }^{1}$ Bruno Juliá-Díaz, ${ }^{1,2}$ Marek Kuś, ${ }^{3}$ and Maciej Lewenstein ${ }^{1,4}$ \\ ${ }^{1}$ ICFO-Institut de Ciències Fotòniques, Parc Mediterrani de la Tecnologia, 08860 Barcelona, Spain \\ ${ }^{2}$ Departament d'Estructura i Constituents de la Matèria, Universitat de Barcelona, 08028 Barcelona, Spain \\ ${ }^{3}$ Center for Theoretical Physics, Polish Academy of Sciences, 02-668 Warszawa, Poland \\ ${ }^{4}$ ICREA-Institució Catalana de Recerca i Estudis Avançats, 08010 Barcelona, Spain
}

(Received 29 May 2013; published 28 August 2013)

\begin{abstract}
A scheme to generate long-range spin-spin interactions between three-level ions in a chain is presented, providing a feasible experimental route to the rich physics of well-known SU(3) models. In particular, we demonstrate different signatures of quantum chaos which can be controlled and observed in experiments with trapped ions.
\end{abstract}

DOI: 10.1103/PhysRevLett.111.090404

PACS numbers: 03.65.Aa, 05.45.Gg, 05.45.Mt

One of the current trends in quantum physics is the quest for controllable quantum many-body systems which can be used as quantum simulators [1,2]. In particular, there is a growing interest in simulating spin and quantum magnetism. In recent years, the focus is moving from $\mathrm{SU}(2)$ spins towards $\mathrm{SU}(N)$ [3,4], which can be realized in earth alkalines, or mixed spin spaces [5]. Here, we show an implementation of SU(3) physics with trapped ions which are known to provide a large degree of control from the experimental point of view.

An important feature of quantum simulators based on ions is the possibility of studying long-range interactions, which are notoriously difficult to simulate classically $[6,7]$. The implementation is based on spin-dependent forces on the ions [8], which have been experimentally achieved recently [9-12]. These interactions lead to new phases, such as exotic forms of superfluidity [13], supersolids [14], quantum crystals, and devil's staircase [15,16].

We concentrate on an important aspect present in SU(3) models: quantum chaos [17,18]. Quantum chaos, as opposed to classical chaos, which can be defined by an exponentially fast growing distance of phase space trajectories, was strongly driven by the understanding of the spectral properties of quantum many-body systems [19]. The large degree of control offered by experiments with ultracold atomic gases has triggered a vast number of experiments to look for different signatures of chaos [20]. Prominent examples are the observation of dynamical tunneling phenomena [21,22], and more recently, the implementation of the kicked-top Hamiltonian on a single atom experiment [23]. Recent proposals look for signatures of chaotic behavior in spin-orbit-coupled condensates [24] or in kicked Bose-Hubbard dimers [25].

In this Letter, we demonstrate that the extremely longrange character of interactions between ions can be used to mimic shell models which are paradigmatic of quantum chaos $[17,18,26]$. We calculate experimentally controllable signatures of chaos and estimate the fidelity of the proposed simulation in the Supplemental Material [27].
Spin-spin interactions of ions.-The main ingredient required to achieve a strong and controllable spin-spin interaction between trapped ions is the implementation of a state-dependent force on the ions. In an early proposal by Mintert and Wunderlich [28], such force is induced by a magnetic field gradient. More flexible proposals provide a force by a Raman coupling of two spin states, which can give rise to phonon excitations. While the proposal by Porras and Cirac [8] uses standing waves in all spatial directions and thereby allows for up to three independent spin-spin interactions, experimental implementations have provided Isingtype spin-spin interactions using a Raman coupling due to a pair of propagating waves with a wave vector difference transverse to the ion alignment $[9,10]$. By choosing the frequency difference between the two lasers close to a vibrational sideband transition, one can enhance and control the phonon excitations and thus the Ising coupling constants. In particular, single vibrational modes can be selected.

Here, we generalize this scheme to three couplings in a system of three-level ions, which allows for an implementation of $\mathrm{SU}(3)$ spin models. All spin states, for instance, those represented by three hyperfine states $|1\rangle \equiv|F=0, m=0\rangle, \quad|2\rangle \equiv|F=1, m=-1\rangle, \quad$ and $|3\rangle \equiv|F=1, m=1\rangle$ in the $S_{1 / 2}$ manifold of $\mathrm{Yb}^{+}$, are coupled as depicted in Fig. 1(a), via off-resonant (a)

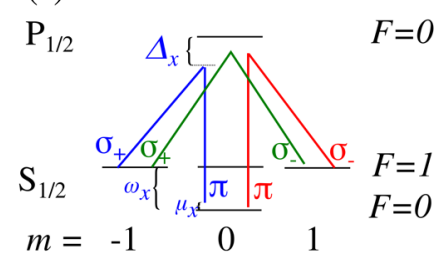

(b)

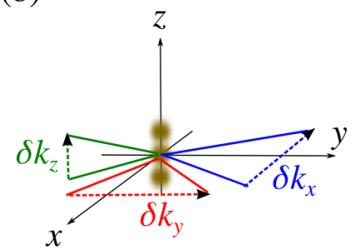

FIG. 1 (color online). (a) Level structure of the ions: Three levels are pairwise coupled by far-detuned Raman lasers, with a beat note $\mu_{\alpha}$ providing a spin-dependent force (see the text). (b) Arrangement of the Raman lasers, with polarizations given in (a) and wave vector difference $\delta k_{\alpha}$ along the axes. 
transitions to an excited state $|e\rangle \equiv|F=0\rangle$ in the $P_{1 / 2}$ manifold. The lasers are arranged as shown in Fig. 1(b), such that each Raman pair has a wave vector difference $\delta \boldsymbol{k}_{\alpha}$ along a different spatial direction $\alpha=x, y, z$. Interference between different couplings can be avoided by choosing different detunings $\Delta_{\alpha}$ from $|e\rangle$.

Each coupling can be equipped with beat notes $\omega_{\alpha} \pm \mu_{\alpha}$, where $\mu_{\alpha}$ describes the detuning from the atomic transition frequency $\omega_{\alpha}$, given by the hyperfine splitting and/or the Zeeman splitting. After adiabatic elimination of the excited state $|e\rangle$ and a rotating wave approximation, the Hamiltonian of each coupling reads [10]

$$
h_{\alpha}(t)=\hbar \sum_{i} \Omega_{\alpha}^{(i)} \sin \left(\mu_{\alpha} t\right) \delta \boldsymbol{k}_{\alpha} \cdot \boldsymbol{x}^{(i)} \tau_{\alpha}^{(i)} .
$$

Here, $\Omega_{\alpha}^{(i)}$ is the two-photon Rabi frequency of the Raman transition $\alpha$ at the position of ion $i$. The spin-flip operators are defined as $\tau_{x} \equiv|1\rangle\langle 2|+$ H.c., $\tau_{y} \equiv|1\rangle\langle 3|+$ H.c., and $\tau_{z} \equiv|2\rangle\langle 3|+$ H.c. Next, we rewrite the position operator in terms of normal coordinates $\delta \boldsymbol{k}_{\alpha} \cdot \boldsymbol{x}^{(i)} \equiv$ $\sum_{m} \eta_{m \alpha}^{(i)}\left(a_{m \alpha} e^{-i \omega_{m \alpha} t}+a_{m \alpha}^{\dagger} e^{i \omega_{m \alpha} t}\right)$, where vibrational modes $m$ along direction $\alpha$ are summed, with $\omega_{m \alpha}, a_{m \alpha}$, and $a_{m \alpha}^{\dagger}$ the corresponding frequency, annihilation, and creation operators. We have introduced the Lamb-Dicke parameter $\eta_{m \alpha}^{(i)}$ characterizing the strength of the spinphonon coupling. For the validity of Hamiltonian (1), it is necessary to have $\eta_{m \alpha}^{(i)} \ll 1$. It is explicitly given by $\eta_{m \alpha}^{(i)}=\sqrt{\hbar / 2 M \omega_{m \alpha}} \mathcal{M}_{m, i}^{\alpha}$. The $N \times N$ matrix $\mathcal{M}_{m, i}^{\alpha}$ are the normal modes in the $\alpha$ direction, found by $\sum_{m^{\prime}} \mathcal{M}_{m, i}^{\alpha} \mathcal{K}_{m m^{\prime}}^{\alpha} \mathcal{M}_{m^{\prime}, i^{\prime}}^{\alpha}=\omega_{m \alpha}^{2} \delta_{i, i^{\prime}}$. The kernel $\mathcal{K}$ contains the Coulomb repulsion and the external trapping of frequency $\omega_{\alpha}$ along each direction. Assuming linearly arranged and equidistant equilibrium positions, it reads

$$
\mathcal{K}_{m, m^{\prime}}^{\alpha}= \begin{cases}\omega_{\alpha}^{2}-c_{\alpha} \sum_{m^{\prime \prime}(\neq m)} \frac{1}{\left|m-m^{\prime \prime}\right|^{3}} & m=m^{\prime} \\ +c_{\alpha} \frac{1}{\left|m-m^{\prime}\right|^{3}} & m \neq m^{\prime},\end{cases}
$$

where $c_{x, y}=1$ and $c_{z}=-2$. We have chosen "ionic" units, in which besides the electric constant $1 /\left(4 \pi \epsilon_{0}\right)$, also the ion mass $M$, the ion charge $q$, and the equilibrium distance $d$ of neighboring ions in the chain are set to unity. Frequencies are then given in units of $\omega_{0} \equiv q /\left(d \sqrt{4 \pi \epsilon_{0} d M}\right)$.

It has been shown in Ref. [10] that the time evolution under the Hamiltonian of Eq. (1) is (to second order in the exponent) given by

$$
U_{\alpha}(t, 0)=\exp \left[\sum_{i} \varphi_{\alpha}^{(i)}(t) \tau_{\alpha}^{(i)}-\sum_{i j} \xi_{\alpha}^{(i, j)}(t) \tau_{\alpha}^{(i)} \tau_{\alpha}^{(j)}\right]
$$

where $\varphi_{\alpha}^{(i)}=\sum_{m}\left[c_{m \alpha}^{(i)}(t) a_{m \alpha}^{\dagger}-\right.$ H.c. $]$ contains a residual spin-phonon coupling, while the second term describes a spin-spin coupling. Both functions $c_{m \alpha}^{(i)}(t)$ and $\xi_{\alpha}^{(i, j)}(t)$ consist of oscillatory terms (with frequencies $\mu_{\alpha}, \mu_{\alpha} \pm \omega_{m \alpha}$ ), which are suppressed by at least one power of $\eta_{m \alpha}^{(i)} \Omega_{\alpha}^{(i)} /\left|\mu_{\alpha}-\omega_{m \alpha}\right| \ll 1$ for sufficiently large detuning from the sideband. In this limit, the dominant contribution to the time evolution stems from a single term in $\xi_{\alpha}^{(i, j)}(t)$ which is linear in $t$, and thus increases constantly. Thus, we can set $c_{m \alpha}^{(i)} \approx 0$ and $\xi_{\alpha}^{(i, j)}(t) \approx i J_{i j}^{\alpha} t$, with

$$
J_{\alpha}^{(i, j)}=\Omega_{\alpha}^{(i)} \Omega_{\alpha}^{(j)} \sum_{m} \frac{\eta_{m \alpha}^{(i)} \eta_{m \alpha}^{(j)} \omega_{m \alpha}}{\mu_{\alpha}^{2}-\omega_{m \alpha}^{2}} .
$$

The time evolution is thus identical to the one of a spin model with spin-spin coupling $J_{\alpha}^{(i, j)}$.

In the presence of more than one coupling, since $\left[h_{\alpha}, h_{\beta}\right] \neq 0$, the time evolution is not simply the product of all $U_{\alpha}$ but consists of additional terms. Up to second order in the Magnus expansion [29], it is equal to $U \simeq\left(\prod_{\alpha} U_{\alpha}\right)\left(\prod_{\alpha \neq \beta} U_{\alpha \beta}\right)$ with $U_{\alpha \beta}=\exp \left\{\sum_{i j} \chi_{\alpha \beta}^{(i, j)}(t) \times\right.$ $\left.\left[\tau_{\alpha}^{(i)}, \tau_{\beta}^{(j)}\right]\right\}$. The functions $\chi_{\alpha \beta}^{(i, j)}$ are given by the integral

$$
\begin{aligned}
\chi_{\alpha \beta}^{(i, j)}= & \sum_{m, n} \int_{0}^{t} d t_{1} \int_{0}^{t_{1}} d t_{2} \sin \mu_{\alpha} t_{1} \sin \mu_{\beta} t_{2} \\
& \times\left(a_{m \alpha} e^{i \omega_{m \alpha} t_{1}}+\text { H.c. }\right)\left(a_{m \beta} e^{i \omega_{m \beta} t_{2}}+\text { H.c. }\right) .
\end{aligned}
$$

For $\mu_{\alpha}=\mu_{\beta}$ and $\omega_{m \alpha}=\omega_{n \beta}$, this function is similar to $\xi_{\alpha}^{(i, j)}(t)$ with small oscillating terms, and one dominant term linear in $t$. However, if couplings $\alpha$ and $\beta$ have different beat notes and/or the mode frequencies are different (for instance, by anisotropic transverse trapping), the linear term disappears, and this contribution can be neglected.

In addition to the spin-spin coupling, a magnetic field term can be generated by a resonant carrier transition $[9,30]$, leading to the effective spin Hamiltonian

$$
H_{\text {spin }}=\sum_{\alpha}\left[\sum_{i=1}^{N} B_{\alpha}^{(i)} \sigma_{z}^{(i)}+\sum_{i \leq j}^{N} J_{\alpha}^{(i, j)} \tau_{\alpha}^{(i)} \tau_{\alpha}^{(j)}\right],
$$

with $\sigma_{z}^{(i)} \equiv|1\rangle\left\langle\left. 1\right|^{(i)}-\mid 3\right\rangle\left\langle\left. 3\right|^{(i)}\right.$. The couplings $J_{\alpha}^{(i, j)}$ given by Eq. (4) can be tuned by the beat notes $\mu_{\alpha}$. In particular, by choosing $\mu_{\alpha}$ sufficiently close to the center-of-mass (c.m.) mode in each direction, $J_{\alpha}^{(i, j)}$ will have only weak dependence on the ion positions $i$ and $j$. Most generally, we assume an inhomogeneous magnetic field $B_{\alpha}^{(i)}$.

Effective $S U(3)$ shell model.-In the limit where $J_{\alpha}^{(i, j)}=$ $J_{\alpha}=$ const $<0$, it is convenient to define spin-flip operators $S_{\sigma \sigma^{\prime}}=\sum_{l=1}^{N}|\sigma\rangle\left\langle\left.\sigma^{\prime}\right|^{(l)}\right.$, acting equally on all spins. Since we have $S_{11}+S_{22}+S_{33}=N$, the $S_{\sigma \sigma^{\prime}}$ provide eight independent operators spanning the SU(3) algebra. For simplicity, we set $J_{\alpha}=J$ and the magnetic field homogeneous. Defining a symmetrized spin operator $\tilde{S}_{\sigma \sigma^{\prime}} \equiv\left(S_{\sigma \sigma^{\prime}}+S_{\sigma^{\prime} \sigma}\right) / \sqrt{2}$, we may rewrite the spin Hamiltonian of Eq. (6) as an ideal model Hamiltonian in terms of these SU(3) operators: 


$$
H_{\text {ideal }}=\frac{B}{\sqrt{2}}\left(\tilde{S}_{11}-\tilde{S}_{33}\right)+J \sum_{\sigma<\sigma^{\prime}} \tilde{S}_{\sigma \sigma^{\prime}} \tilde{S}_{\sigma \sigma^{\prime}}
$$

Besides the replacement $S_{\sigma \sigma^{\prime}} \rightarrow \tilde{S}_{\sigma \sigma^{\prime}}$, this Hamiltonian is identical to the three-level Lipkin-Meshkov-Glick (LMG) Hamiltonian [31], a model where particles can occupy three different shells with single-particle energies $-B, 0$, and $B$. Two-body interactions of particles in the same shell lead to pair tunneling into the other shells. The LMG Hamiltonian has applications in nuclear physics, and its three-level version is particularly appealing as a not fully integrable spin model in the context of quantum chaos [18,32].

Our Hamiltonian (7) differs from the LMG Hamitonian only by an additional interaction $\sum_{\sigma \neq \sigma^{\prime}} S_{\sigma \sigma^{\prime}} S_{\sigma^{\prime} \sigma}$. Because of its SU(3) symmetry and the fact that this additional term is a Casimir operator of SU(3) [18], the Hamiltonian (7) and the LMG Hamiltonian are fully equivalent: Having a block-diagonal structure with respect to different representations of SU(3), in each block, the additional interaction simply reduces to a constant.

Apart from particle exchange symmetry, the LMG model has a second symmetry [32]: As particles can change the spin state only pairwise, the occupation numbers of each spin state $\left\langle S_{11}\right\rangle,\left\langle S_{22}\right\rangle$, and $\left\langle S_{33}\right\rangle$ can only change by two and thus are fixed to either even $(e)$ or odd $(o)$ values. This gives rise to four signature classes $e e e$, $o e o, o o e$, and $e o o$ for $N$ even, or ooo, eeo, eoe, and oee for $N$ odd. This signature class symmetry is also present in Eq. (6), that is, in the model with a space-dependent coupling $J_{\alpha}^{(i, j)}$ given by Eq. (4). On the other hand, the spin exchange symmetry is lost. Still, there is invariance under parity, as $\boldsymbol{J}^{(i, j)}=\boldsymbol{J}^{(N-i)(N-j)}$, due to the parity invariance of $\mathcal{K}$. For a numerical diagonalization of this Hamiltonian, it is convenient to construct the eigenbasis of parity and signature class. While the Fock states are already signature eigenstates, a combination of at most two Fock states also yields a parity eigenstate.

Quantum chaos in the LMG model.-In the classical limit of the three-level LMG model, its phase space can be divided into regions of chaotic and regular motion [32]. Accordingly, the quantum model also shows signatures of both chaotic and regular behaviors. While in chaotic quantum systems the spectrum features level repulsion, regular behavior is related to level clustering. These features are nicely displayed by the unfolded distribution [17] of the level spacings $s$ in the spectrum. A Poisson distribution $P(s)=e^{-s}$ indicates level clustering, while chaotic Hamiltonians with time-reversal invariance follow a Wigner distribution $P(s)=(\pi / 2) s \exp \left[-\pi s^{2} / 4\right]$. In Ref. [32], it has been shown for the LMG model that one part of the spectrum is spaced according to the Poisson distribution, while another part follows a Wigner spacing.

This results in a level spacing distribution as shown in Fig. 2(a) for $N=10$ and a magnetic field $B=\left\langle J_{\alpha}^{(i, j)}\right\rangle / 2$.
We have used the realistic Hamiltonian (6), with a trap frequency $\omega_{\alpha}=0.1 \omega_{0} / \delta_{\alpha}$ at a relative detuning from the c.m. mode $\delta_{\alpha}=\left(\omega_{\text {c.m. } \alpha}-\mu_{\alpha}\right) / \omega_{\text {c.m. } \alpha}>0$. As is shown in the Supplemental Material [27], this Hamiltonian reproduces with high fidelity the physics of the ideal model; see Eq. (7). We have unfolded the spectrum separately in each symmetry block of the Hamiltonian (that is, for fixed parity and signature class). The level spacing distribution is found to be broader than the Wigner distribution and has its maximum shifted towards smaller spacings. This suggests to consider the Brody distribution $P_{q}(s)$ which interpolates between the Wigner $(q=1)$ and the Poisson distribution $(q=0)[33,34]$ :

$$
P_{q}(s)=\alpha(q+1) s^{q} \exp \left[-\alpha s^{q+1}\right],
$$

with $\alpha=\{\Gamma[(q+2) /(q+1)]\}^{q+1}$. The value of $q$ provides a measure of the degree of chaoticity in the system. As shown in Fig. 2(a), our distribution is well represented by $q=0.7$.

The behavior expressed by these statistics can be illustrated by representing the evolution of a few energy levels when one parameter of the Hamiltonian is changed, e.g., the magnetic field strength $B$. In each symmetry block, we find both level crossings and avoided level crossings, as already expected from the level spacing distribution. In Fig. 2(b), we illustrate, for $N=4$, a part of the spectrum where all level crossings belonging to states of the same symmetry (parity) are avoided. Of course, the crossings between states of different parity are not avoided. In Fig. 2(c), we then show the same part of the spectrum in the presence of an additional small magnetic field gradient $\delta_{B}=0.2$, that is, for an inhomogeneous magnetic field $B_{\text {inhom }}(x)=B+\delta_{B} x$. This contribution breaks the parity symmetry, turning the previously symmetry allowed level crossings [Fig. 2(b)] into avoided ones.
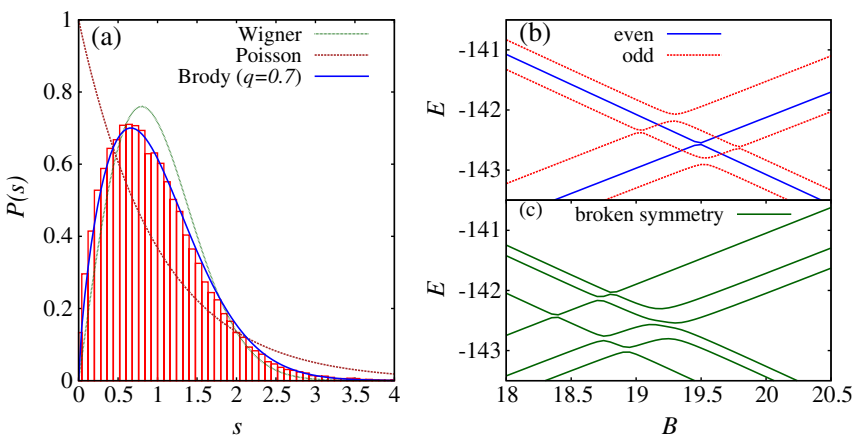

FIG. 2 (color online). (a) Level spacing distribution for $N=10$ after unfolding the spectrum separately in each symmetry block of the Hamiltonian. (b) Avoided crossing of energy levels with equal parity symmetry, exemplified for $N=4$ in the eoe signature class upon tuning the magnetic field strength $B$. (c) The same as in (b) but in the presence of a small additional magnetic field gradient $\delta_{B}=0.2$ breaking the parity symmetry. This leads to avoided crossings of all energy levels. 
Experimental detection of quantum chaos.-The signatures of quantum chaos discussed so far are hard to measure in a system of trapped ions. More easily, quantum chaos can be detected by preparing the system initially in a coherent quantum state and then observing the subsequent time evolution of this state [21-23]. Unitarity of quantum evolution prevents a definition of quantum chaos mirroring the usual one in classical systems: exponential sensitivity to initial conditions. Instead, for quantum-chaotic motion, it is argued that a relevant signature is provided by high sensitivity of the time evolution onto slight changes in the Hamiltonian parameters [17].

In order to relate our study of the quantum dynamics to its classical limit, the initial states will be $\mathrm{SU}(3)$ coherent spin states defined as $\left|z_{1}, z_{2}\right\rangle \equiv \mathcal{N} \exp \left[z_{1} S_{31}+z_{2} S_{21}\right]|0\rangle$, where $|0\rangle$ denotes a Fock state which is fully spin polarized in the lower spin component $|0\rangle \equiv|11 \cdots 1\rangle$, and $\mathcal{N}$ normalizes the state. The complex parameters $z_{1}$ and $z_{2}$ define the classical state in terms of four canonical variables $q_{1}, q_{2}, p_{1}$, and $p_{2}$. The classical Hamiltonian is then given by [32]

$$
H_{\text {class }}\left(q_{1}, q_{2}, p_{1}, p_{2}\right)=\lim _{N \rightarrow \infty}\left\langle z_{1}, z_{2}\left|H_{\text {ideal }} / N\right| z_{1}, z_{2}\right\rangle .
$$

We have performed the classical time evolution using a Runge-Kutta method. The coherent states with small average energy are mostly found to have regular behavior, while states of intermediate energy behave rather chaotically.

We will now search for signatures of chaos in the quantum time evolution, driven by the Hamiltonian $H_{\text {spin }}$. We again choose $\omega_{\alpha}=0.1 \omega_{0} / \delta_{\alpha}$ and consider two initial states: one in a classical regular region and the second one in a classical chaotic region. As shown in Fig. 3(a), a minimal change of $1 \%$ in the parameter $B$ has little effect on the quantum time evolution of the regular state compared to its effect on the evolution of the chaotic state. This indicates that even for a system of eight ions, far from the classical limit, we observe clear signatures of quantumchaotic behavior in correspondence with the expected behavior in the classical limit. For comparison, the figure also shows the time evolution of the same initial states for a Hamiltonian where by choosing $J_{x}=J_{y}=0$, one spin state has been dynamically frozen. In this way, the model reduces to an SU(2) LMG model [31], which is integrable, and accordingly shows no trace of quantum chaos.

While the overlaps shown in Fig. 3(a) are not directly accessible in experiments, signatures of chaotic behavior can also be found in the evolution of a spin component of the state. In our scheme, the occupation of the level $|m=0\rangle$ can be measured with high precision by resonantly exciting ions from this level and observing the subsequent fluorescence. For a system prepared in the regular state, a regular pattern is expected, while an erratic pattern is a signature of chaotic motion [17]. We exemplify this in Figs. 3(b) and 3(c), showing the time evolution of $\left\langle S_{11}\right\rangle$
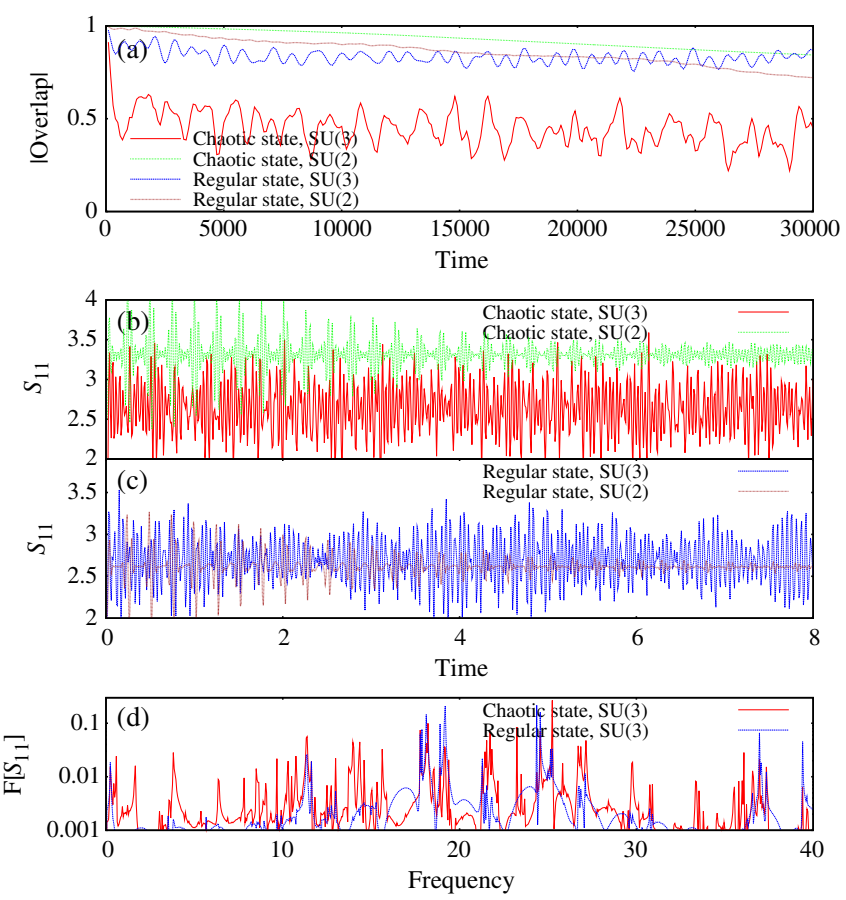

FIG. 3 (color online). (a) We evolve one chaotic state $z_{1}=$ $-0.10+0.61 i$ and $z_{2}=-0.83+0.26 i$ and one regular state $z_{1}=-1.06+0.26 i$ and $z_{2}=-1.04+0.33 i$, with $N=8$ particles in the Hamiltonian $H_{\text {spin }}\left(B, J_{x}, J_{y}, J_{z}\right)(6)$ for $B=0.5$ and $B=0.505$. For each initial state, we plot the overlap between the evolved states for the two $B$ 's as a function of time. The curves labeled with $\mathrm{SU}(3)$ are obtained by choosing all interactions $J_{\alpha}$ to be transmitted by equally strong forces $K_{\alpha}$, while the SU(2) curves are obtained for $J_{x}=J_{y}=0$. (b), (c) For the same states as in (a) and with $B=0.5$, we plot the occupation number $\left\langle S_{11}\right\rangle$ as a function of time. (d) The Fourier transform of the SU(3) curves in (b) and (c).

for the regular and the chaotic states given above, evolved with $B=0.5$ in the full $\mathrm{SU}(3)$ Hamiltonian and in the reduced SU(2) Hamiltonian. The curves for SU(2) clearly show a regular pattern, while in the $\mathrm{SU}(3)$ case, the differences between the chaotic and the regular states are less obvious. We therefore perform a Fourier analysis of these curves after subtracting its average and normalizing the amplitude of the oscillation. In the Fourier spectrum, shown in Fig. 3(d), the regular evolution is dominated by only a few peaks, while the spectrum of the chaotic evolution is much more diversified.

Summary.-We have presented a scheme to realize SU(3) spin models with trapped ions. The spin-spin interaction parameter is tunable, allowing the simulation of spin models with long-range interactions. In this Letter, we have focused on the situation where interactions are almost constant with respect to the position of the ions. Then, the systems describe a three-level LMG model which interpolates between quasi-integrable and chaotic dynamics. Chaos on the quantum level is usually characterized in terms of the spectral statistics, which demands 
high-precision measurements of many energy levels, hardly possible in the settings similar to the one discussed in our Letter. Instead, we propose a feasible scheme to detect quantum chaos in the dynamics of the model. Our proposal thus provides a powerful experimental tool to study the onset and signatures of chaos in quantum systems, based on state-of-the-art techniques.

This work has been supported by the EU (NAMEQUAM, AQUTE), ERC (QUAGATUA), Spanish MINCIN (FIS2008-00784 TOQATA), Generalitat de Catalunya (2009-SGR1289), Polish NSC Grant No. DEC-2011/02/A/ST1/00208, and Alexander von Humboldt Stiftung. B. J. D. is supported by the Ramón y Cajal Program.

[1] M. Lewenstein, A. Sanpera, and V. Ahufinger, Ultracold Atoms in Optical Lattices-Simulating Quantum ManyBody Systems (Oxford University Press, New York, 2012).

[2] M. Lewenstein and V. W. Liu, Nat. Phys. 7, 101 (2011).

[3] A. V. Gorshkov, M. Hermele, V. Gurarie, C. Xu, P. S. Julienne, J. Ye, P. Zoller, E. Demler, M. D. Lukin, and A. M. Rey, Nat. Phys. 6, 289 (2010).

[4] D. Banerjee, M. Bögli, M. Dalmonte, E. Rico, P. Stebler, U.-J. Wiese, and P. Zoller, Phys. Rev. Lett. 110, 125303 (2013).

[5] P. A. Ivanov and F. Schmidt-Kaler, New J. Phys. 13, 125008 (2011).

[6] T. Koffel, M. Lewenstein, and L. Tagliacozzo, Phys. Rev. Lett. 109, 267203 (2012).

[7] P. Hauke and L. Tagliacozzo, arXiv:1304.7725.

[8] D. Porras and J. I. Cirac, Phys. Rev. Lett. 92, 207901 (2004).

[9] A. Friedenauer, H. Schmitz, J. T. Glueckert, D. Porras, and T. Schaetz, Nat. Phys. 4, 757 (2008).

[10] K. Kim, M.-S. Chang, R. Islam, S. Korenblit, L.-M. Duan, and C. Monroe, Phys. Rev. Lett. 103, 120502 (2009).

[11] J. W. Britton, B. C. Sawyer, A. C. Keith, C.-C. J. Wang, J. K. Freericks, H. Uys, M. J. Biercuk, and J. J. Bollinger, Nature (London) 484, 489 (2012).

[12] R. Blatt and C.F. Roos, Nat. Phys. 8, 277 (2012).

[13] B. Capogrosso-Sansone, C. Trefzger, M. Lewenstein, P. Zoller, and G. Pupillo, Phys. Rev. Lett. 104, 125301 (2010).
[14] P. Anders, P. Werner, M. Troyer, M. Sigrist, and L. Pollet, Phys. Rev. Lett. 109, 206401 (2012).

[15] F. J. Burnell, M. M. Parish, N. R. Cooper, and S. L. Sondhi, Phys. Rev. B 80, 174519 (2009).

[16] P. Hauke, F. M. Cucchietti, A. Müller-Hermes, M.-C. Bañuls, J. I. Cirac, and M. Lewenstein, New J. Phys. 12, 113037 (2010).

[17] F. Haake, Quantum Signatures of Chaos (Springer, New York, 2001).

[18] S. Gnutzmann, F. Haake, and M. Kuś, J. Phys. A 33, 143 (2000).

[19] O. Bohigas, M. J. Giannoni, and C. Schmit, Phys. Rev. Lett. 52, 1 (1984).

[20] M. Raizen and D. A. Steck, Scholarpedia 6, 10468 (2011).

[21] W. K. Hensinger, H. Häffner, A. Browaeys, N.R. Heckenberg, K. Helmerson, C. McKenzie, G. J. Milburn, W. D. Phillips, S. L. Rolston, and H. Rubinsztein-Dunlop et al., Nature (London) 412, 52 (2001).

[22] D. A. Steck, W. H. Oskay, and M. G. Raizen, Science 293, 274 (2001).

[23] S. Chaudhury, A. Smith, B. E. Anderson, S. Ghose, and P. S. Jessen, Nature (London) 461, 768 (2009).

[24] J. Larson, B. M. Anderson, and A. Altland, Phys. Rev. A 87, 013624 (2013).

[25] C. Khripkov, D. Cohen, and A. Vardi, Phys. Rev. E 87, 012910 (2013).

[26] S. A. Gardiner, J. I. Cirac, and P. Zoller, Phys. Rev. Lett. 79, 4790 (1997).

[27] See Supplemental Material at http://link.aps.org/ supplemental/10.1103/PhysRevLett.111.090404 for an estimate of errors in the quantum simulation.

[28] F. Mintert and C. Wunderlich, Phys. Rev. Lett. 87, 257904 (2001).

[29] S. Blanes, F. Casas, J. Oteo, and J. Ros, Phys. Rep. 470, 151 (2009).

[30] K. Kim, M. S. Chang, S. Korenblit, R. Islam, E. E. Edwards, J. K. Freericks, G. D. Lin, L. M. Duan, and C. Monroe, Nature (London) 465, 590 (2010).

[31] H. Lipkin, N. Meshkov, and A. J. Glick, Nucl. Phys. 62, 188 (1965)

[32] D. C. Meredith, S. E. Koonin, and M. R. Zirnbauer, Phys. Rev. A 37, 3499 (1988).

[33] T. A. Brody, Lett. Nuovo Cimento 7, 482 (1973).

[34] D. Engel, J. Main, and G. Wunner, J. Phys. A 31, 6965 (1998). 